\title{
Neural network model of decision making in electric power facilities under conditions of uncertainty
}

\author{
Isomiddin Siddikov ${ }^{1}$ and Oksana Porubay $^{1 *}$ \\ ${ }^{1}$ Department of Information Processing and Management, Tashkent State Technical University \\ named after Islam Karimov, 100000 Tashkent, Uzbekistan
}

\begin{abstract}
The article is devoted to the issue of creating a mathematical model of the problem of making management decisions in electric power facilities based on modern intelligent technologies, which makes it possible to take into account the influence of various factors on the operating modes of the power system. A systematic approach to describing processes in the mathematical language of the theory of fuzzy sets is proposed. To solve the problem of controlling the operating modes of the power system, a neurofuzzy network has been developed that combines the algorithms of TakagiSugeno fuzzy inference, as well as a recurrent neural network. An adaptive learning algorithm based on the Frechet method is proposed for training a neural network. The analysis of the efficiency of the fuzzy control model under the conditions of various modes of functioning of the local power system is carried out.
\end{abstract}

\section{Introduction}

Electric power systems (hereinafter referred to as EPS), as elementary parts of the infrastructure of the national economy, have strong and diverse connections with various industries, it can also be noted that to a large extent, they determine the living conditions of the population, the state of the natural environment, etc.

In power generation control systems (PGCS), such systems are widely used, which are based, in particular, on mathematical models built using deterministic and random methods $[1,2,3]$.

Such methods are based on assumptions about the accuracy, purpose, and limitations of the initial data, and the possible uncertainty "tender" is closed in the Procrustean bed of interval estimates (parameters of the normal distribution) $[1,3]$.

As you know, in electric power systems (EPS), continuous technological processes are carried out continuously, and calculations and assessment of the state at the current time are possible only in discrete modes of individual current values of their interval parameters, without taking into account the structure, which leads to a confusing situation and frequent loss of possible solutions. In this regard, the problem of incompleteness and inaccuracy of the initial data arises, which requires constant complication of mathematical models.

* Corresponding author: oksanaporubay@gmail.com 
Nevertheless, the existing mathematical models for managing electricity production have proven themselves positively, especially when simulating steady-state processes under "normal" conditions, such as assessing the state of an EPS, analyzing reliability, calculating power based on economic indicators, planning modes, and so on [1, 4].

As a result of these difficulties, it is necessary to develop the developed ETSs, which are designed to provide methods for semantic analysis of situations and allow evaluating and solving the following tasks:

1) operations not only with individual numerical values of the EPS state parameters but also with sets of permissible values, beams of disturbed motion trajectories, system constraints;

2) maximum consideration of constraints and multi-dimensional assumptions in the assumptions of a set of circumstances based on privilege constraints, adapted to the dynamics of the process, inaccessible to individuals in the management process;

3) mapping of current processes to a set of linguistic variables and implementation of controls or recommendations for managing states in a form that includes both numerical and linguistic variables;

4) linguistic description of processes and development of methods for their implementation in the practice of operational management of energy systems, research, and identification of optimal relationships between sets of numerical and linguistic variables [5].

Therefore, the simplest approach is based on the ideas of expert systems in combination with the methods of the theory of fuzzy sets. The practice of using them for diagnosing the state of complex systems has shown fairly high efficiency. Productivity is exceptional and is achieved by methods of fuzzy set theory in the management of complex dynamical systems. The tandem of these areas together creates a broad methodological basis for the development of a system of semantic analysis (SSA) of dynamic processes in energy objects. However, implementation will require the additional formation of the structure of SSA structures and the principles of their operation.

\section{Method}

Reviewing various methods, one can apply the systematic approach to the description of mathematical theories proposed by N. Bourbaki $[6,7]$. Consider a decision-making system based on fuzzy set theory in the following mathematical structure:

$$
S=<P, X, L, M, U, R>,
$$

where: $P$ - many parameters describing the state of energy objects;

$X=B\left(x_{p}\right)-$ the set of subsets of numerical values of parameters from $P$;

$L$ - many linguistic (verbal) meanings of $P$;

$x\left(l_{j}\right) \in X-$ a subset of the definition of linguistic meaning $l_{j} \in L$;

$M$ - the set of mappings $x$ on $[0,1]$ functions $\mu\left(x_{i}\right)$, characterizing the degree of membership of $x_{i} \in X$ to $x\left(l_{j}\right)$ for $l_{j} \in L$;

$U$ - many control solutions;

$R$ - many relations of the form $L \times L, L \times U, U \times U$.

Example: We have a set $P$, consisting of one parameter - the current frequency, then the set $X$ can be specified by the interval [8-11], and the set $L$ by the following values:

$l_{1}=\left\langle\right.$ low» $\mathrm{c} x\left(l_{1}\right)=[0.0,50.0]$ and 


$$
\begin{gathered}
\mu\left(x_{i}\right)=\left\{\begin{array}{l}
\left(50,0-x_{i}\right) \in[49,50] ; \\
0, x_{i} \geq 50 ; \\
1, x_{i}<49 ;
\end{array}\right. \\
l_{2}=\text { «normal»c } x\left(l_{2}\right)=[49.5,50.5] \text { and } \\
\mu\left(x_{i}\right)=\left\{\begin{array}{l}
2 \times\left(x_{i}-49.5\right), x_{i} \in[49.5,50,0] ; \\
2 /\left(50.5-x_{i}\right) \in[50.0,50.5] ; \\
0, x_{i} \in[49.5,50.5] ;
\end{array}\right. \\
l_{3}=\left\langle\text { high»c } x\left(l_{30}\right)=[50.0,+\infty]\right. \text { and } \\
\mu\left(x_{i}\right)=\left\{\begin{array}{l}
\left(x_{i}-50.0\right) x_{i} \in[50,51] ; \\
0, x_{i} \geq 51 ; \\
1, x_{i}<50 ;
\end{array}\right.
\end{gathered}
$$

The type of the membership function is determined in the process of interpreting the results obtained, but the results of the study $[1,5,7]$ show that sufficient solutions are obtained using linear or exponential relationships. Any current numerical value of the "frequency of current" parameter can be indicated with its various linguistic meanings.

For example, for $x=49.8$ we get $\mu\left(l_{i}\right)=\{0.2,0.6,0.0\}$.

Let the set $U$ include control decisions:

$u_{1}=$ «hierarchical consumer disconnection»;

$u_{2}=$ «monitoring the current state»;

$u_{3}=$ «power regulation».

And let the ratio $R=L \times U$ be characterized by the matrix:

$$
R=\left\{r_{i j}\right\}=
$$

\begin{tabular}{|l|l|l|l|}
\hline & $\mathrm{u}_{1}$ & $\mathrm{u}_{2}$ & $\mathrm{u}_{3}$ \\
\hline $\mathrm{l}_{1}$ & 0.8 & 0.4 & 0.2 \\
\hline $\mathrm{l}_{2}$ & 0.3 & 0.9 & 0.4 \\
\hline $\mathrm{l}_{3}$ & 0.6 & 0.3 & 0.8 \\
\hline
\end{tabular}

Then the control solution of our example can be determined by the following parameters:

$$
u=\arg \max \left\{\max \min \mu_{x}\left(l_{i}\right), r_{i j}\right\}, u_{k} \in U \quad u_{j} \in U \quad l_{i} \in L
$$

At the current moment in time, the decision maker (hereinafter referred to as the decision maker) must exercise "control over the current state" with a degree of confidence of 0.6.

To solve the problems of managing regional energy consumption processes, a fuzzy neural network was chosen, which combines the Takagi-Sugeno fuzzy inference algorithm and a return (repetitive) neural network. Such a structure allows, on the one hand, to give the undefined approximator better opportunities in the context of poorly formalized functions, and, on the other hand, to significantly increase the flexible properties of the neural network.

As a mathematical forecasting model, a model of the form (6) was chosen, in which the dynamic equation is formed for successive moments, and the transition matrix from the moment of time $k$ to the moment of time $(k+1)$, is a function of the moment of the cycle and is different during the period of 


$$
X_{k+1}=F(k) X_{k}+\xi_{F},
$$

where the matrix $F(k)$ is set for each moment (or for certain sections) of the load graph, $\xi_{F}$ - accounting vector of an arbitrary component.

In the case of the Takagi-Sugeno fuzzy inference algorithm, the structure of I / O rules can be represented as:

$$
R^{k}: \text { if a }(t-1) \text { there is } \mathrm{X}_{1}^{k}, x(t-r) \text { there is } X_{r}^{k} \text {, then } y(t)=a_{0}^{k}+\sum_{i=1}^{r} a_{1}^{k} y(t-1), k=\overline{1, k}
$$

where $R^{k}$ - set of rules for functioning; $x(t)=\left(x_{1}(t), \ldots, x_{m}(t)\right)$ - vector of input variables; $y(t)$ - output vector; $X_{1}^{k}, X_{m}^{k}, Y^{k}$ - range of values corresponding to input and output values; $a^{k}$ - constant quantity (constant) that represents the correspondence of the output quantity to the weighted influence of the input quantities. Membership terms are of Gaussian type (gaussmf), output terms are linear (linear).

When evaluating the quality of network training, it is proposed to use the objective function in the form of the sum of the squared differences between the actual and expected values of the output signals. Then, for a certain number of $(j=1,2, \ldots p)$ training samples $(x, e)$, the objective function can be defined as follows::

$$
E(w)=\frac{1}{2} \sum_{j=1}^{p} \sum_{m=1}^{M}\left(y_{m}^{(j)}-e_{m}^{(j)}\right)^{2},
$$

where $m$ - number of network outputs;

$j$ - number of learning samples fed to the network input;

$y_{m}^{(j)}$ - network output at step $j$;

$e_{m}^{(j)}$ - required network output at step $j$;

$E(w)$ - target function of neural network.

An important stage in the formation of a fuzzy neural network is the process of its training, which consists of finding the optimal version of the accuracy of the network operation while minimizing the parameter $E$ (8). Since the function $E$ can have an arbitrary shape, learning as a whole should be considered as a problem of multiextremal nonconvex optimization.

The goal of the learning algorithm is to adjust the weights of the links $\alpha_{c}$ in such a way as to minimize the loss function $E\left(y_{1}, \ldots, y_{n}, u_{1}, \ldots u_{n}\right)$, which in turn is a function of the system errors

The training of neural networks is to minimize the error of the control system. This is done by adjusting such a neural network parameter as the link weights $\alpha_{k}$.

If the system is described by equation (9), then the bond weights $\alpha_{k}$ are adjusted according to a certain rule:

$$
\alpha_{K}=F_{i n K}^{\prime}\left[x_{i n K}\right] \cdot\left(\frac{y_{\text {out } K}}{y_{\text {inK }}}\right) \sum_{S \in Q_{\text {out }}} \alpha_{S} \cdot \alpha_{S}-\gamma \cdot F_{\text {inK }}^{\prime}\left[x_{i n K}\right] \cdot y_{\text {out } K} \cdot \frac{\delta E}{\delta y_{\text {inK }}},
$$

where $\gamma>0$ - learning rate coefficient;

$F_{i n K}^{\prime}\left[x_{i n K}\right]$ - Frechet derivative;

$E-$ loss function (error) $k \in K$. 
Provided that equation (9) has a unique solution for the value of $\alpha_{k}$, in which the loss function $E\left(y_{1}, \ldots, y_{n}, u_{1}, \ldots u_{n}\right)$ is uniformly decreasing in time, the following equality will be satisfied:

$$
\alpha_{k}=-\gamma \frac{\delta E}{\delta \alpha_{k}}, k \in K
$$

With this approach, the neural network can be broken down into its constituent elements, which are presented in the form of an elementary neural network (Figure 1).

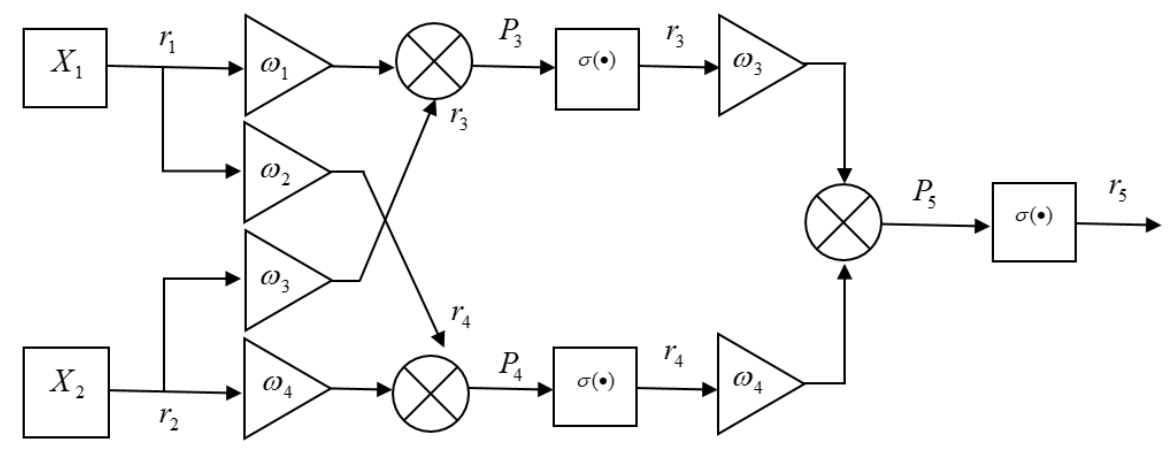

Fig. 1. Block diagram of an elementary neural network.

The mathematical neural network learning algorithm will be considered in the form:

$$
\begin{aligned}
& P_{n}=\sum_{s \in D n} \omega_{s} \cdot r_{\text {pres }} \\
& r_{n}=\sigma(p n),
\end{aligned}
$$

where $n$ - neuron index; $s$ - synapse index; $D n$ - set of neuron input synapses $n$; pres and posts - presynaptic and postsynaptic neuron corresponding to a synapse $s ; \omega_{s}$ synapse weight; $p n$ - membrane potential of a neuron $n ; r_{n}$ - neuron firing frequency $n$; $\sigma$ - the activation function of the sigmoid type, which is presented in the following form:

$$
\sigma(x)=\frac{1}{1+e^{-x}}
$$

In this case, the weight of synapses can be determined by the formula:

$$
\omega_{s}=r_{\text {pres }}\left(\varphi_{\text {posts }} \sigma\left(-P_{\text {posts }}\right)+\gamma \cdot f_{\text {posts }}\right) \text {, }
$$

where $\varphi_{m}=\sum_{S \in A_{n}} \omega_{s} \cdot \omega_{s}, \gamma$ - forward feedback coefficient for all neurons, $\varphi_{n}$ - the forwardreverse error signal.

It should be noted that the described learning algorithm is equivalent to the error repropagation algorithm, but the re-propagation neural network is not required to transfer the error from network outputs to its input.

\section{Results and Discussions}

Analysis of the performance of a fuzzy neural network when implementing two different optimization methods (hybrid) - which is a combination of least squares and a method of reducing the inverse gradient using the Sugeno algorithm, and (backpropo) - 
backpropagation of an error for a network of the same structure, when using the input term dsigmf a network with the same structure showed the best quality of performance, while the first method (hybrid) showed itself the best (Figure 2). Its results: the average error was $2.1 \%$; the training time was $8.5 \mathrm{~s}$., in the opposite case, the indicators of the second method (backpropo) (Figure 3) were $3.174 \%$ and 17.6 s. respectively. As a result of the implementation of the proposed neural network forecasting model, the forecast time (monthly with hourly division) was $43.7 \mathrm{~s}$. In this case, the error turned out to be less than $2.22 \%$.

\begin{tabular}{|c|c|c|c|c|c|c|c|}
\hline \multicolumn{7}{|l|}{1.2} & 250 \\
\hline \multicolumn{7}{|l|}{1} & 200 \\
\hline \multicolumn{2}{|l|}{0.6} & \multicolumn{5}{|c|}{0.8} & 150 \\
\hline \multicolumn{7}{|l|}{0.4} & 100 \\
\hline \multirow{3}{*}{$\begin{array}{r}0.2 \\
0\end{array}$} & & & & & & & 50 \\
\hline & psigmf & trimf & trapmf & gaussm & pimf & dsigmf & 0 \\
\hline & \multicolumn{6}{|c|}{ Input Term Type Accessories } & \\
\hline Training sample E & 0.011 & 0.035 & 0.024 & 0.017 & 0.015 & 0.012 & \\
\hline Training sample $\varepsilon n$ & 0.054 & 0.123 & 0.089 & 0.095 & 0.077 & 0.073 & \\
\hline Training sample $\mathrm{r}$ & 0.983 & 0.934 & 0.956 & 0.971 & 0.983 & 0.981 & \\
\hline Training sample $\varepsilon \%$ & 2.103 & 4.381 & 4.218 & 3.847 & 3.478 & 2.1 & \\
\hline $\begin{array}{c}\text { Number of learning } \\
\text { epochs }\end{array}$ & 61 & 98 & 197 & 188 & 195 & 63 & \\
\hline Training time, sec. & 7.2 & 5.1 & 6.3 & 6.1 & 6.9 & 8.5 & \\
\hline
\end{tabular}

Fig. 2. Graph of comparative analysis of the algorithm for the implementation of models based on a fuzzy neural network (hybrid)

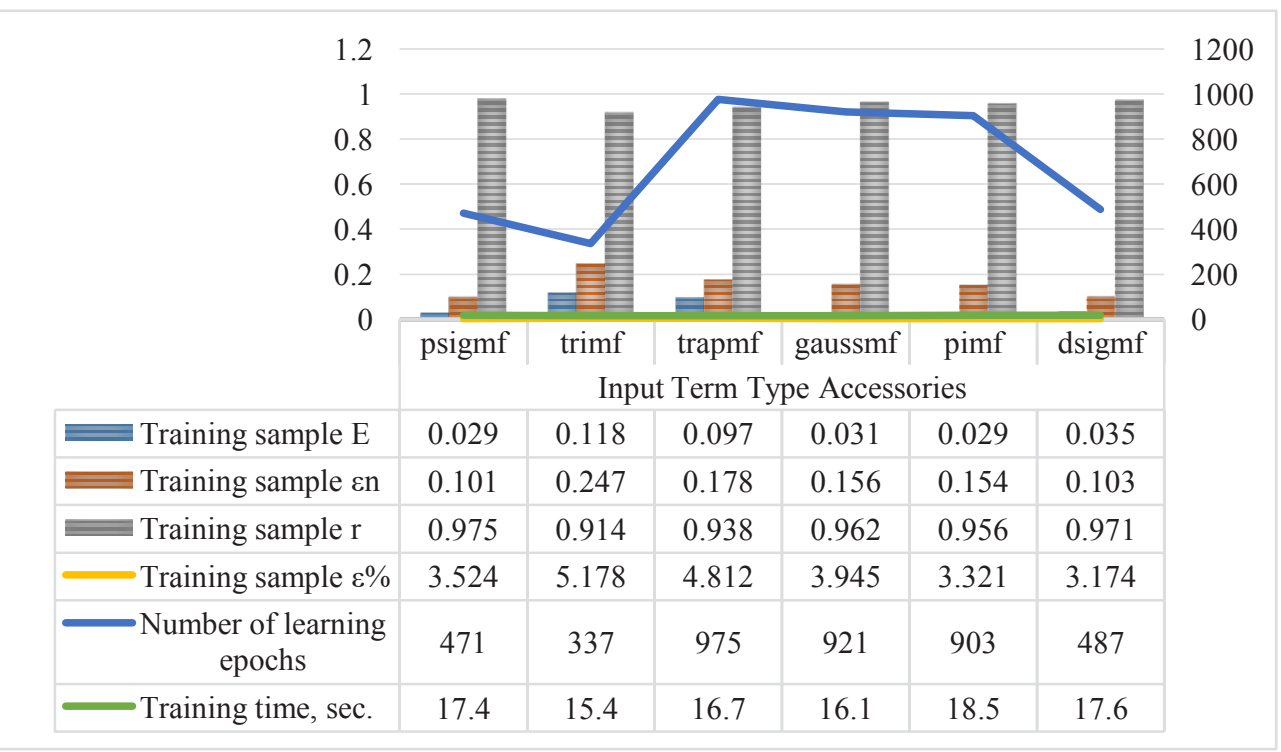

Fig. 3. Training graph of a fuzzy neural network - Sugeno (backpropo) 


\section{Conclusions}

An algorithm for tuning a fuzzy neural network has been developed, which is characterized by the possibility of using data obtained as a result of the implementation of a variety of alternative procedures for training a neural network model.

The analysis of the efficiency of the fuzzy control model in the context of various operating modes of the local power system is carried out.

To predict the level of energy consumption, a software package was developed and tested, in real conditions. The developed software is applicable for the intellectualization of regional power supply management processes based on a fuzzy neural network and a fuzzy control model, which can integrate with appropriate tools that provide automatic dispatching, which is distinguished by systems tested in real conditions, and facilitates the management of energy supply organizations.

\section{References}

1. M. Munasinghe, Engiineering - Economic Analysis of Electric Power System, IEEE 72(4), 28-72 (1984)

2. M.G. Morgan, S.N. Talukdar, Electric Power Load Management: Some Technical, Economic, Regulatory and Social Issues, IEEE 67(2) 241 - 312 (1979)

3. B.F. Wollenberg, T. Sakaguchi, Artificial Intelligence in Power System Operations. IEEE 75(12), 1678 - 1685 (1987)

4. A. Ali Kadhem, N.I. Abdul Wahab, I. Aris, J. Jasni, A.N. Abdalla, Reliability Assessment of Power Generation Systems Using Intelligent Search Based on Disparity Theory, Energies 10(3), 343 (2017)

5. S.A. Orlovsky, Decision-making problems with fuzzy initial information, Science, Moscow (1989)

6. L. Corry, Nicolas Bourbaki and the Concept of Mathematical Structure, Synthese 92(3), 315-348 (1992).

7. K.S. Pister, Mathematical modeling for structural analysis and design, Nuclear Engineering and Design 18(3), 353-375 (1972)

8. I.Kh. Siddikov, O.V. Porubay, M.V. Lazareva, A.A. Abdulkhamidov, Trends in the development of intelligent systems when making management decisions in Uzbekistan, Universum: technical sciences 2(71), 10-14 (2020)

9. I.Kh. Siddikov, D.M. Umurzakova, Mathematical Modeling of Transient Processes of the Automatic Control System of Water Level in the Steam Generator, Universal Journal of Mechanical Engineering 7(4), 139-146 (2019)

10. I.Kh. Siddikov, D.M. Umurzakova, Synthesis Algorithm for Fuzzy-logic Controllers, 14th International IEEE Scientific and Technical Conference Dynamics of Systems, Mechanisms and Machines, Dynamics, Omsk, Russia (2020)

11. I.Kh. Siddikov, D.M. Umurzakova, Neuro-fuzzy Adaptive Control system for Discrete Dynamic Objects, International Conference on Information Science and Communications Technologies Applications, Trends and Opportunities, Tashkent, Uzbekistan (2019) 